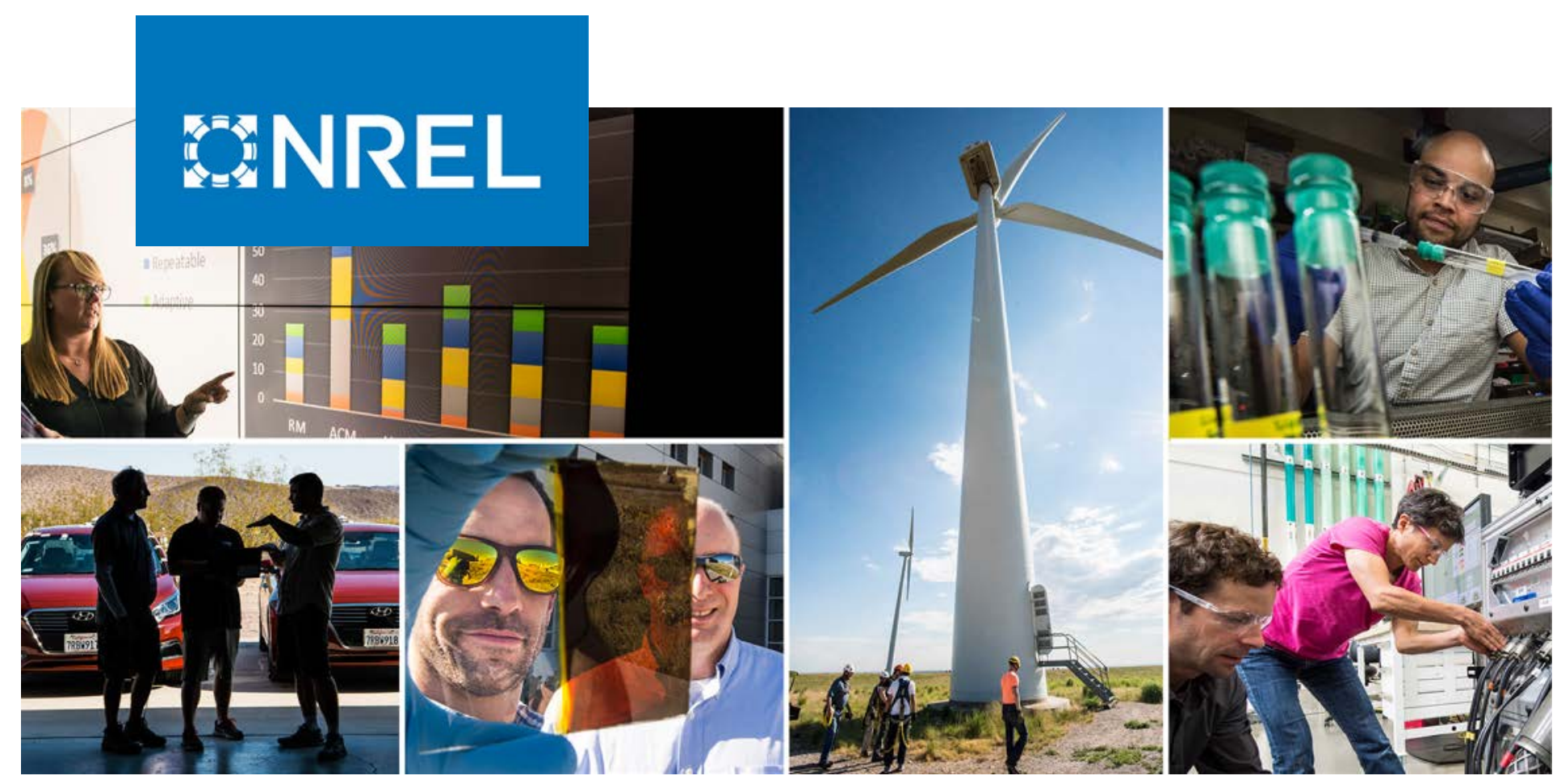

Performance Evaluation of an Advanced Distributed Energy Resource Management Algorithm

\title{
Preprint
}

Jing Wang, Jeff Simpson, Rui Yang, Bryan Palmintier, Soumya Tiwari, and Yingchen Zhang

National Renewable Energy Laboratory

Presented at the 2021 IEEE International Conference on Communications, Control, and Computing Technologies for Smart Grids (SmartGridComm) October 25-28, 2021

NREL is a national laboratory of the U.S. Department of Energy Office of Energy Efficiency \& Renewable Energy

Operated by the Alliance for Sustainable Energy, LLC

This report is available at no cost from the National Renewable Energy Laboratory (NREL) at www.nrel.gov/publications.
Conference Paper

NREL/CP-5D00-80109

January 2022 


\title{
BNREL
}

\section{Performance Evaluation of an Advanced Distributed Energy Resource Management Algorithm}

\section{Preprint}

\author{
Jing Wang, Jeff Simpson, Rui Yang, Bryan Palmintier, \\ Soumya Tiwari, and Yingchen Zhang \\ National Renewable Energy Laboratory
}

\author{
Suggested Citation \\ Wang, Jing, Jeff Simpson, Rui Yang, Bryan Palmintier, Soumya Tiwari, and Yingchen \\ Zhang. 2022. Performance Evaluation of an Advanced Distributed Energy Resource \\ Management Algorithm: Preprint. Golden, CO: National Renewable Energy Laboratory. \\ NREL/CP-5D00-80109. https://www.nrel.gov/docs/fy22osti/80109.pdf.
}

\begin{abstract}
(c) 2022 IEEE. Personal use of this material is permitted. Permission from IEEE must be obtained for all other uses, in any current or future media, including reprinting/republishing this material for advertising or promotional purposes, creating new collective works, for resale or redistribution to servers or lists, or reuse of any copyrighted component of this work in other works.
\end{abstract}

NREL is a national laboratory of the U.S. Department of Energy Office of Energy Efficiency \& Renewable Energy Operated by the Alliance for Sustainable Energy, LLC

This report is available at no cost from the National Renewable Energy Laboratory (NREL) at www.nrel.gov/publications.

Contract No. DE-AC36-08GO28308
Conference Paper

NREL/CP-5D00-80109 January 2022

National Renewable Energy Laboratory 15013 Denver West Parkway Golden, CO 80401 303-275-3000 • www.nrel.gov 


\section{NOTICE}

This work was authored by the National Renewable Energy Laboratory, operated by Alliance for Sustainable Energy, LLC, for the U.S. Department of Energy (DOE) under Contract No. DE-AC36-08GO28308. Funding provided by the U.S Department of Energy Office of Energy Efficiency and Renewable Energy Solar Energy Technologies Office Agreement Number 32962. The views expressed herein do not necessarily represent the views of the DOE or the U.S. Government.

This report is available at no cost from the National Renewable Energy Laboratory (NREL) at www.nrel.gov/publications.

U.S. Department of Energy (DOE) reports produced after 1991 and a growing number of pre-1991 documents are available free via www.OSTI.gov.

Cover Photos by Dennis Schroeder: (clockwise, left to right) NREL 51934, NREL 45897, NREL 42160, NREL 45891, NREL 48097, NREL 46526.

NREL prints on paper that contains recycled content. 


\title{
Performance Evaluation of an Advanced Distributed Energy Resource Management Algorithm
}

\author{
Jing Wang, Jeff Simpson, Rui Yang, Bryan Palmintier, Soumya Tiwari, Yingchen Zhang \\ Power Systems Engineering Center, National Renewable Energy Laboratory Golden, CO 80401, USA \\ Jing.Wang@nrel.gov, Yingchen.Zhang@nrel.gov
}

\begin{abstract}
This paper presents performance evaluation of a new distributed energy resource management system (DERMS) algorithm via an advanced hardware-in-the-loop (HIL) platform. The HIL platform provides realistic testing in a laboratory environment, including the accurate modeling of sub-transmission and distribution networks, the DERMS software controller, and 84 power hardware solar photovoltaic (PV) inverters, standard communication protocols, and a capacitor bank controller. The DERMS algorithm is also called, Grid-Optimization of Solar (GO-Solar) platform which includes predictive state estimation (PSE) and online multiple objective optimization (OMOO) to dispatch the legacy devices and distributed energy resources (e.g., PV). The voltage regulation performance is evaluated under three scenarios, volt-var smart inverter (baseline), and DERMS control for $100 \%$ and $30 \%$ of $\mathrm{PV}$. The results show that controlling $30 \%$ of PV systems with the GO-Solar platform may provide the best balance of control performance and implementation cost.
\end{abstract}

Index Terms-distributed energy resource management system (DERMS), hardware-in-the-loop (HIL), online multiobjective optimization (OMOO), voltage regulation.

\section{INTRODUCTION}

With the proliferation of DERs in distribution systems, many utilities face challenges integrating large numbers of non-utility devices into operations at all levels and ensuring grid stability and corresponding controls [1]. Industry and IEEE working groups have worked together to address DER integration by identifying and standardizing the functions and operation requirements that individual DERs can perform autonomously, such as frequency-watt and voltage-volt ampere reactive (volt-var) controls [2]. However, in many situations, significantly improved performance can be achieved with centralized coordination of distribution assets using advanced distribution management systems (ADMS) or other feeder-wide coordination systems such as distributed energy resource management systems (DERMS), or the coordination between them [3].

ADMS is a software platform commonly used by utilities to integrate numerous utility systems [4], including supervisory control and data acquisition (SCADA), geographic information system, outage management system, etc. An ADMS can provide a suite of operation functionalities

This work was supported by Alliance for Sustainable Energy, LLC, the manager and operator of the National Renewable Energy Laboratory for the U.S. Department of Energy (DOE) under Contract No. DE-AC3608GO28308. This material is based upon work supported by the U.S Department of Energy's Office of Energy Efficiency and Renewable Energy (EERE) under Solar Energy Technologies Office (SETO) Agreement Number 32962. The views expressed in the article do not necessarily represent the views of the DOE or the U.S. Government. The U.S. Government retains and the publisher, by accepting the article for publication, acknowledges that the U.S. Government retains a nonexclusive, paid-up, irrevocable, worldwide license to publish or reproduce the published form of this work, or allow others to do so, for U.S. Government purposes. such as volt-var optimization. DERMS is an emerging technology that bridges the gap between larger system coordination (e.g., ADMS) and potentially large numbers of DER devices to aggregate, monitor, and control groups of DERs to provide a more manageable set of services [5]. Additional research and evaluation is needed to further advance DERMS and ensure that the systems work as expected before field deployment. Therefore, a generic test bed to evaluate the coordinated control among ADMS, other utility management systems (e.g., DERMS), DERs, and legacy utility equipment controllers was developed in [6]. This testbed provides a realistic laboratory test environment, including real-time co-simulation of full-scale distribution systems provided by utility partners, controller and power hardware, and industry standard communications protocols.

Reference [7] leverages the co-simulation capability developed in [6] and validates one function of the DERMS (also called Grid Optimization of Solar (GO-Solar) platform) with limited number of hardware DER inverters. This work continues the effort in [7] and we expanded the effort to evaluate full functions of the GO-Solar platform and test it with large scale power HIL (PHIL) evaluation (84 hardware devices) and one legacy device (the capacitor bank controller) under multiple realistic scenarios. The HIL setup and evaluation in this paper provides a valuable framework and guidance in a realistic laboratory environment to test futuristic control and operation systems that manage large penetration of solar and other DERs.

\section{OVERVIEW OF THE HIL SETUP}

This hardware-in-the-loop (HIL) platform validates the optimization and control algorithm of the GO-Solar platform against a simulated real-world distribution system, using data provided by the Hawaiian Electric Company. The overall diagram of the setup is presented in Figure 1. The main elements of the HIL platform includes co-simulation, the GOSolar Platform (PSE and F-OMOO), the PHIL (84 DER inverters, grid simulators, sensors, and PV emulators), the communication interface, and the capacitor bank controller.

For the co-simulation, the sub-transmission is modelled in OpenDSS with a time step of $4 \mathrm{~s}$ and the whole distribution feeder in OPAL-RT in ePhasorSim with a time step of $0.005 \mathrm{~s}$. Data are exchanged between OpenDSS and OPAL-RT to close the co-simulation loop and allow the two simulation tools to synchronize. The GO-Solar Platform receives measurements from the co-simulation and sends back the optimized real and reactive power (PQ) set points to the DERs simulated in OPAL-RT in real time. The hardware DER inverters also receives the PQ set points from the GO-Solar platform, through the communication interface. One capacitor bank controller is included and evaluated as controller HIL (CHIL), which receives the voltage set points dispatched by 
the F-OMOO. This voltage set point is passed through OPALRT and sent to the capacitor bank controller through analog output. OpenDSS, the GO-Solar platform and the communication interface are implemented in a laptop using Hierarchical Engine for Large-scale Infrastructure CoSimulation (HELICS) framework [8]. More detailed implementation of the HELCIS framework is shown in Figure 2. In this setup, HELICS is the key player in this integrated platform to merge all the elements (software and hardware), synchronize them, allocate the execution time of each element and transfer data between elements.

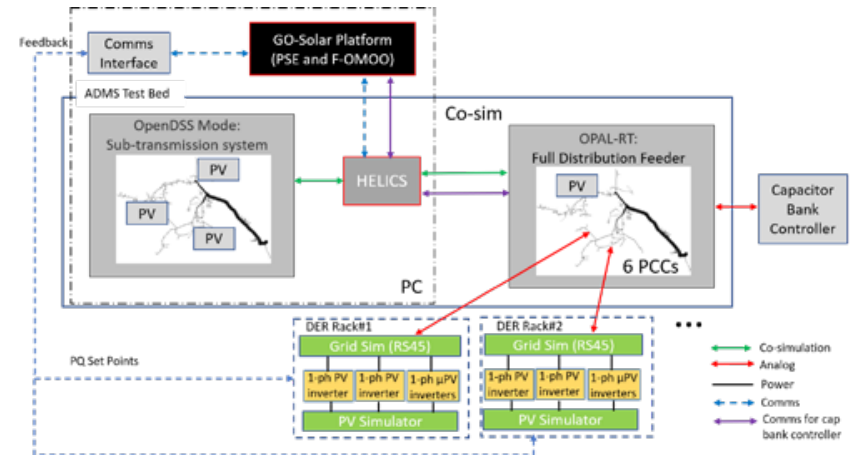

Figure 1. Overall diagram of the integrated HIL platform. PSE stands for predictive state estimation, and F-OMOO means fast-scale online multiobjective optimization.

The structure of the HELICS co-simulation for the HIL implementation is shown in Figure 2. There are five agents in this framework, including the OpenDSS, Opal-RT, OMOO, state estimation, and communication agents. HELICS acts as an integrator to synchronize all the agents together and allow each agent run with its own time step. The execution time step for each agent is $4 \mathrm{~s}$ for OpenDSS, $0.1 \mathrm{~s}$ for OPAL-RT, $30 \mathrm{~s}$ for $\mathrm{OMOO}, 60 \mathrm{~s}$ for state estimation, and $30 \mathrm{~s}$ for the Communication (interface to the hardware inverter). The data exchanged between agents are marked in one particular color. For instance, the measurements for the state estimation agent are $V_{\text {meas }}$, and $P Q_{\text {meas }}$, marked in green. Note that the laptop running HELICS machine needs to communicate with the OPAL-RT target and all the DER hardware inverters. So, they need to be in the same local area network (LAN). Since the OPAL-RT and all DER hardware inverters use different LAN, an internet extension is made in the HELICS machine to allow the computer communicates with OPAL-RT with one LAN, and allow the computer communicates with all DER hardware inverter with a different LAN.

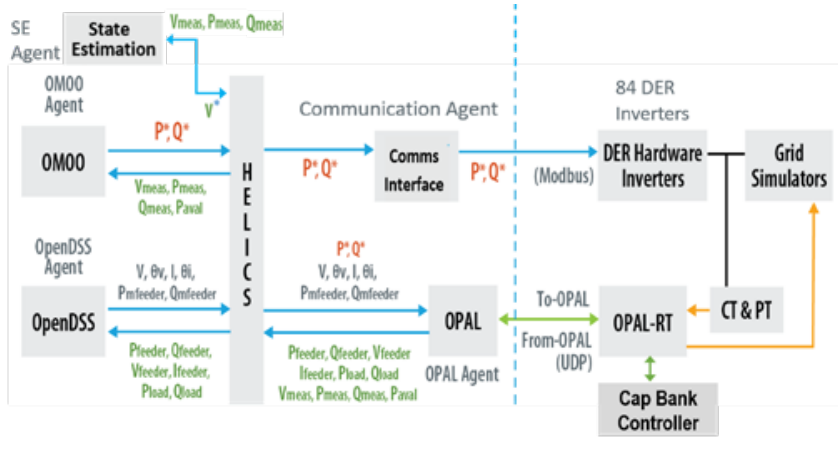

Figure 2. Schematic diagram of the HELICS architecture.

\section{DESCRIPTION OF EACH ELEMENT}

Since this paper is an extension of [7] and some elements are already presented, only additional contents are described in this section.

\section{A. Co-simulation Platform}

As shown in Fig. 1, the sub-transmission system is simulated in OpenDSS and the whole distribution feeder is simulated in ePhasorSim in OPAL-RT. We leverage the NREL ADMS testbed capability to implement the cosimulation [6]. Generic code is developed in OpenDSS and OPAL agents to allow the co-simulation of the two software tools. The changes need to be made are the name of the interconnected feeder/branch, and corresponding measurements (e.g., active and reactive power, voltage magnitude and phase angle). Once customized changes are made, these two agents can run together with OPAL-RT to test the co-simulation of the network by comparing the measured voltage and phase angle of feeder head in OPAL-RT, and the measured active and reactive power of the feeder head in OpenDSS against their reference values. If those measurements are close to their reference values, the cosimulation is considered complete and stable.

\section{B. Grid Solar Platform}

The objective of the GO-Solar platform is to provide a software platform that gives utility companies the capability to seamlessly dispatch both legacy devices (at the distribution and sub-transmission levels) and DERs to achieve systemwide performance and reliability targets - such as reducing voltage violation. A key innovation of this DERMS is to manage system-wide control objectives using only a small percentage of field devices. This enables much larger scaling with greatly reduced communications infrastructure costs. To do so, a high-precision, machine-learning based predictive state estimator (PSE) is being developed to enable sparse data to provide system-wide awareness and short-term forecasting. The PSE drives online multi-objective optimization (OMOO) algorithms to allow for the fast dispatch of only a small number of DERs such as solar PV through smart inverters while asynchronously dispatching legacy devices such as voltage regulators and capacitor banks to avoid the need to control millions of devices in real time.

The high-level control architecture of the GO-Solar platform is presented in Figure 3. The PSE takes heterogeneous measurements collected from limited locations in the system to first estimate the system voltages at the current time step using matrix completion method and then forecast the system voltages in the short-term future using a multikernel learning method. The forecasted system voltages are then fed into an OMOO algorithm which determines the optimal setpoints of various controllable devices-including both the fast-responding DERs and legacy devices - in the short-term future by solving an optimal power flow problem. The optimal setpoints for the predicted short-term future are then communicated with each device. In real time, the OMOO is employed to follow the plan produced by the PSE and adjust the setpoints of DERs to cope with the fast variability of DERs and optimize the voltage profile in the system. The detailed problem formulation and algorithm of the GO-Solar platform can be found in [9]. 


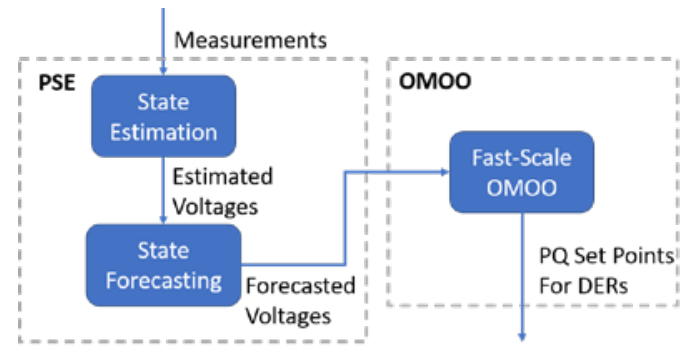

Figure 3. The control architecture of the GO-Solar platform.

\section{DER Assets and Power Hardware-in-the-Loop}

The voltage regulation performance of the GO-Solar Platform is validated using a large number of hardware DER inverters through PHIL. This allows evaluating the real response of hardware inverters dispatched by the GO-Solar Platform, while also assessing the stability and performance of the GO-Solar platform. Figure 1 and 2 show the actual implementation in the lab in a high-level view, where all the hardware DER inverters are interfaced with HELICS and connected with supporting equipment, such as grid simulators, sensors, and PV emulators.

Table 1 lists all the PHIL DER inverters of each point of common coupling (PCC), and the total physical and simulated capacity for each PCC. PCCs \#1-4 have the same physical capacity of $11.84 \mathrm{kVA}$, while PCCs \#5-6 are rated at $9.84 \mathrm{kVA}$. In the electrical network simulations, this physical capacity is scaled to a higher different simulated capacity for each PCC. To achieve the simulated capacity in OPAL-RT, scaling down is performed in Communication agent to ensure that the dispatched set points for the hardware DER inverters are not higher than rated capacity. Likewise, scaling up is performed in OPAL-RT to ensure that the lumped power at each PCC can represent the higher simulated active and reactive power. The ratio of scaling up is the reciprocal of the ratio of scaling down for each PCC.

Table 1 List of PHIL DER inverters of each PCC

\begin{tabular}{|c|c|c|c|c|}
\hline Rack & \# Devices & Physical Devices & $\begin{array}{c}\text { Total } \\
\text { capacity }\end{array}$ & $\begin{array}{c}\text { Simulated PV } \\
\text { capacity }\end{array}$ \\
\hline PHIL-1 & 14 & (1) $3 \mathrm{kVA} \mathrm{PV}$, (1) $5 \mathrm{kVS} \mathrm{PV}$, (12) $320 \mathrm{VA} \mu \mathrm{PV}$ & $11.84 \mathrm{kVA}$ & $23.5 \mathrm{kVA}$ \\
\hline PHIL-2 & 14 & (1) $3 \mathrm{kVA} \mathrm{PV}$, (1) $5 \mathrm{kVA} \mathrm{PV,} \mathrm{(12)} 320 \mathrm{VA} \mu \mathrm{PV}$ & $11.84 \mathrm{kVA}$ & $19 \mathrm{kVA}$ \\
\hline $\begin{array}{l}\text { PHIL-3 } \\
\end{array}$ & 14 & (1) $3 \mathrm{kVA} \mathrm{PV}$, (1) $5 \mathrm{kVA} \mathrm{PV,} \mathrm{(12)} 320 \mathrm{VA} \mu \mathrm{PV}$ & $11.84 \mathrm{kVA}$ & $93.9 \mathrm{kVA}$ \\
\hline PHIL-4 & 14 & (1) $3 \mathrm{kVA} \mathrm{PV}$, (1) $5 \mathrm{kVA} \mathrm{PV}$, (12) $320 \mathrm{VA} \mu \mathrm{PV}$ & $11.84 \mathrm{kVA}$ & $67.6 \mathrm{kVA}$ \\
\hline PHIL-5 & 14 & (1) $3 \mathrm{kVA} \mathrm{PV}$, (1) $3 \mathrm{kVA} \mathrm{PV}$, (12) $320 \mathrm{VA} \mu \mathrm{PV}$ & $9.84 \mathrm{kVA}$ & $119.2 \mathrm{kVA}$ \\
\hline PHIL-6 & 14 & (1) $3 \mathrm{kVA} P \mathrm{PV}$, (1) $3 \mathrm{kVA} P \mathrm{PV}$, (12) $320 \mathrm{VA} \mu \mathrm{PV}$ & $9.84 \mathrm{kVA}$ & $54 \mathrm{kVA}$ \\
\hline Total & 84 & 6 PCCs & & \\
\hline
\end{tabular}

Figure 5 presents the hardware connection for one DER rack. The simulated voltage of the PCC in OPAL-RT is sent to the grid simulator to reconstruct the physical voltage. This nominal voltage is $219 \mathrm{~V}$, and it is scaled up to $240 \mathrm{~V}$ for the single-phase DER inverter. This single-phase transformer is also an isolation transformer which protects the grid simulator from the potential hazards or damages coming from the DER rack. On the other hand, the transformer also electrically decouples the grid simulator and the DER inverters connected at each DER rack, and three PCCs connected at the phase A, $\mathrm{B}$ and $\mathrm{C}$ of the grid simulator. This is particularly important for the PHIL hardware setup using one grid simulator to support three independent PCCs that are located at different places in the distribution feeder. After the transformer, a junction box which has fuse installed and a common PCC connection with multiple plugs for DER inverter connections is developed. The AC side of the DER inverters are connected at the junction box's common connection, and the DC side of the DER inverters are connected as following: the SMA PV inverter and the Fronius PV inverter are supplied by the PV emulator, and the SunPower micro-PV inverters are supplied by a DC power supply with a fixed DC voltage $(60 \mathrm{~V})$ and current $(100 \mathrm{~A})$. Note that 12 SunPower micro-PV inverters are paralleled connected, and the total power references $\left(P^{*}\right.$ and $\left.Q^{*}\right)$ are divided by 12 and then sent to each of the micro$\mathrm{PV}$ inverter. The lumped current at the PCC and the PCC voltage are measured using a CT and PT located at the secondary side of the isolation transformer, respectively. These two measurements are then sent back to OPAL-RT using analog input channels. To interface six PCCs with hardware DER inverter connected, six analog output channels and 12 analog input channels are mapped in OPAL-RT. Ideal transformer method is used to interface the hardware inverters with the controlled current source in OPAL-RT, and the calculated power is used to drive the current source and replicate the dynamics of power at each PCC.

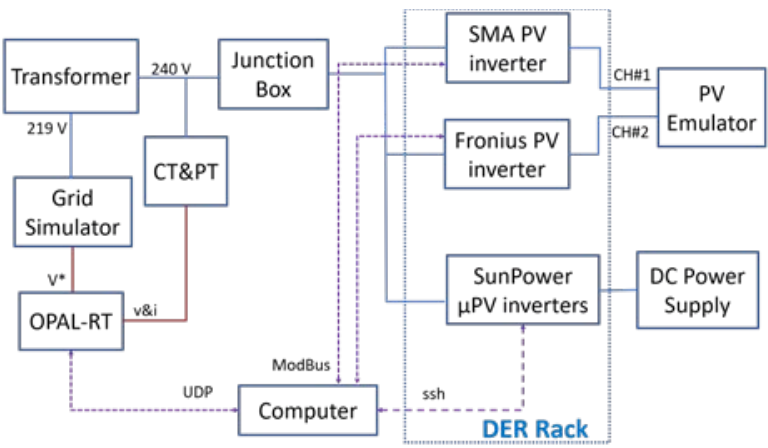

Figure 4. The hardware setup and connection for each DER rack.

\section{Communication Interface}

In this HIL setup, the HELICS machine where the hierarchical control algorithm is embedded dispatches the SMA PV inverter and Fronius PV inverter using ModBus communication protocol, and dispatches SunPower micro-PV inverters using a special communication protocol, secure shell protocol (ssh). A communication agent is developed in the HELICS platform to work as an interface between the OMOO agent and the hardware inverters as shown in Figure 2. The total time needed to dispatch all inverters and receive feedback from them is tested using the communication agent log file which records the time stamp of each execution step. Based on the time stamps of a 2-hour run, the exact execution time of all steps are calculated, and an average of the time steps is used as the time step of the communication agent.

The communication agent defines the communication interface functions of the ModBus and ssh, the Internet Protocol (IP) address and settings of all the inverters (e.g., time out error and master/slave mode) [10]. The active and reactive power references for each DER rack are passed from the grid optimization control OMOO agent to the communication agent. Then the active and reactive power references are scaled down to the capacity of each DER rack, and further divided proportionally for each inverter based on the rated capacity. Next, the Communication agent calls the ModBus/SSH interface functions, reaches each hardware inverter through the IP address of the inverter, writes the registers for the active and reactive power references, and reads the registers for the active and reactive power measurements. 


\section{E. Capacitor Bank Controller Hardware-in-the-Loop}

Figure 5 (a) and (b) shows the setup of the capacitor bank controller and the schematic diagram of interface in OPALRT, respectively. The voltage reference for the capacitor bank controller is low voltage analog output signal $( \pm 16 \mathrm{~V})$, which is amplified by the OMICRON amplifier to the level of 120 $\mathrm{V}$. This high voltage is used to drive the Beckwith Capacitor bank controller which compares this voltage with its upper $(126 \mathrm{~V})$ and lower $(114 \mathrm{~V})$ voltage limits. By default, the capacitor bank is closed. If the voltage reference is higher than the upper limit, the "open" signal will be "high", otherwise, the "open" signal is kept "low". Similar, if the voltage reference is lower than the lower limit, the "close" signal will be "high", otherwise, the "close" signal is kept "low". These two signals are sent back to the OPAL-RT through analog inputs, and a control logic is developed in OPAL-RT to determine the final control signal of the circuit breaker of the simulated capacitor bank. This is illustrated in Figure 5 (b) which also explains the data processing of the reference voltage for the capacitor bank. The reference voltage of the capacitor bank is in RMS value, which is then reconstructed into sinusoidal waveform, scaled down to low voltage signal, and output through analog output to the OMICRON amplifier. This CHIL setup can showcase the performance of the OMOO with the capability of dispatching legacy device.
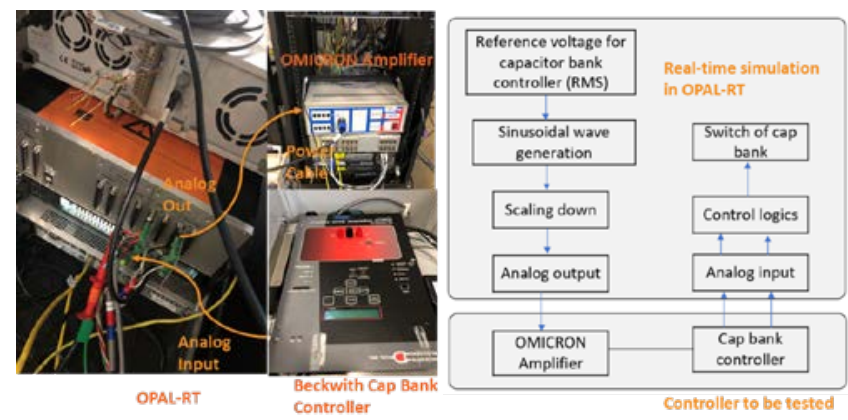

Figure 5. (a) The capacitor bank controller setup and (b) schematic diagram in OPAL-RT.

\section{EVAluation OF THE GO-SOlar PlatForm}

To evaluate the voltage regulation performance of the GOSolar platform, all of the PV units were first operated as smart inverters with volt-VAR functions to provide a baseline scenario. Then, the GO-Solar Platform is evaluated when controlling $100 \%$ of PV inverters and then when only controlling $30 \%$ of PV inverters to test how well the GO-Solar Platform performs with a limited amount of DERs under controlled. This section describes the configuration and setup for these three scenarios. Note that the baseline scenario is evaluated in OPAL-RT through software-based real-time simulation, while the GO-Solar scenarios are evaluated through HIL simulation using the setup shown in Figure 1.

\section{A. Baseline Scenario}

The volt-VAR smart inverter function used in this work is presented in Figure 6. This is a standard curve used in existing smart PV inverters. The VAR priority is implemented for each PV inverter. Therefore, if there is insufficient power due to the lack of sunlight, the reactive power is generated first and then the rest of available power is for active power. For the realtime implementation in OPAL-RT, the smart inverter function is triggered every $1 \mathrm{~s}$ with the simulation runs every $5 \mathrm{~ms}$. A deadband of 0.005 p.u. is added for the two slopes (0.95-0.97 and 1.03-1.05) so that the reactive power can maintain the same if the change in voltage is smaller than the band. This helps improve the stability of the smart inverter function.

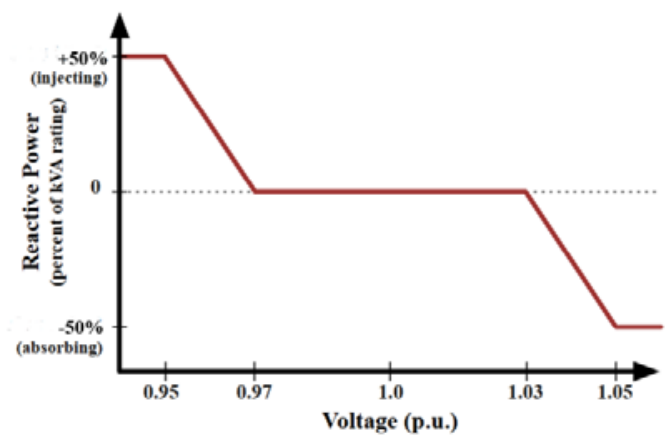

Figure 6. Volt-VAR cure of smart inverter function [11].

\section{B. GO-Solar Platform Controls 100\% PV Inverters}

In this scenario, the GO-Solar platform is included and evaluated with all the PVs being controlled, the capacitor bank controller and six DER racks with 84 DER hardware inverters are tested. For the F-OMOO, the upper and lower target voltages are set to be 1.035 and 0.96 p.u. which is comparable to the smart inverter function response limits $(\geq 1.03$ and $\leq 0.95$ ). All the measurements from the PV inverters (active and reactive power, and available active power) are sent to the F-OMOO algorithm which computes and sends the optimal power setpoints for the PV units. Six out of 211 PV inverters are simulated in hardware, and the rest are simulated in OPAL-RT ePhasorSim. In addition, the capacitor bank controller receives the voltage setpoint from State Estimation, and the open/close signal is sent back to OPAL-RT to control the simulated capacitor bank. This scenario assumes the GOSolar platform has the full controllability and visibility of all the PV inverters.

\section{GO-Solar Platform Controls $30 \%$ PV Inverters}

This scenario is developed based on the previous one, only $30 \%$ PVs are controlled or dispatched by the F-OMOO, and the rest of the PVs operate in unity power factor mode. Those controlled PVs are selected based on their dual variable, mu, a control parameter output of F-OMOO [10]. A higher mu means a higher need for voltage regulation. This is an important criterion to select which inverter is being controlled and can help future field deployments identify the most important or economic PVs to control to manage to the cost of communication and control infrastructures.

To implement $30 \%$ PVs being controlled, less data (measurements and control signals for PV inverters) is exchanged between the OPAL Agent and the OMOO Agent. For simplicity, the data mapping dictionary exchanged through HELICS is kept the same, but only $30 \%$ of the measurements are fed into the OMOO control algorithm. Then even though the control set points for all PV inverters are sent back to through HELICS, only those for the $30 \%$ of PVs that are controllable are used by OPAL-RT, with the other PV inverters configured to provide all available active power. Note that DER Rack \#2 is not controlled, and the available power is used as the active power reference (reactive power reference is zero), and the rest of DER Racks are dispatched by the OMOO agent. There is no change needed for the Communication Agent. 


\section{EXPERIMENTAL RESULTS}

This section presents the evaluation results for the voltage regulation performance of the GO-Solar platform under the three scenarios defined in Section IV. For the evaluation, a 2$\mathrm{h}$ simulation window from 12:00 p.m.-14:00 p.m. is selected since it includes smooth solar irradiance in the first hour and fluctuating solar irradiance in the second hour. This enables evaluating the GO-Solar performance for both clear and cloudy conditions in a single run. The load and PV profiles are presented in Figure 7 (a), which shows that the power generated by PV is higher than the active power demand. Figure 7 (b) shows the over voltage violations of the system without any controls in PVs.
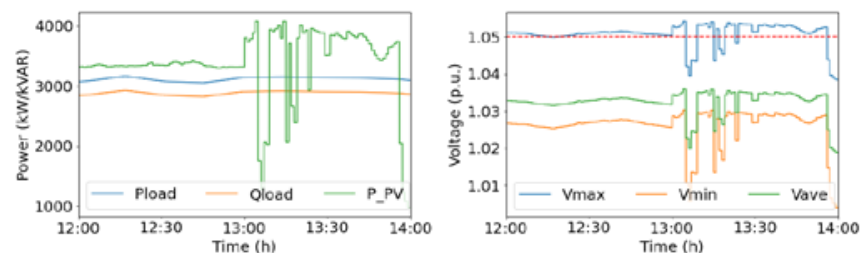

Figure 7. (a) Load and PV profiles used for the evaluation and (b) system voltages with all PV units operating in unity power factor.

System Voltage Measurements: Figure 8 shows the system maximum, average, and minimum voltages for the three scenarios: the baseline (volt-VAR), full PV control (100\%), and 30\% PV control). It shows similar responses for the min, max, and average system voltages for two GO-Solar scenarios. The voltage performance with all PVs under control is somewhat better; but there is still noticeable improvement with $30 \%$ PVs under control compared to the baseline. This is particularly true for average and the minimum system voltages. For the maximum voltage, the GO-Solar platform with $30 \%$ PVs under control still exhibits better performance most of the time. Overall, Figure 8 indicates superior voltage regulation performance of the GO-Solar platform (both $30 \%$ and $100 \%$ ) comparing to the volt-VAR function.
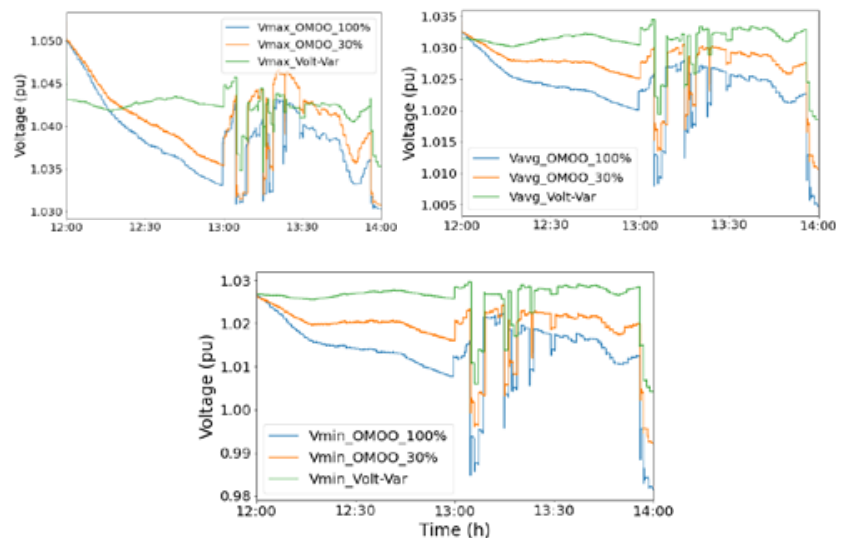

Figure 8. The (a) maximum, (b) average, and (c) minimum of system voltages with PV units operating in volt-VAR smart inverter function and controlled by F-OMOO.

Total PV Outputs: Figure 9 shows the total active and reactive power generation of all PVs. For the active power output, the four curves overlap, and the active power curtailment levels are: $0.08 \%$ for volt-var, $0.4 \%$ for $100 \% \mathrm{PV}$ controlled with GO-Solar, and $0.24 \%$ for $30 \%$ PV controlled with GO-Solar. For the reactive power output, the $30 \%$ GOSolar case has smaller reactive power flow than the $100 \%$ control case, but larger reactive power use than the baseline. All three approaches adjust the reactive power outputs of PVs to respond to system high voltages. However, the GO-Solar platform (both $30 \%$ and $100 \%$ ) can respond more dynamically to system changes (e.g., caused by the solar irradiance) for voltage regulation. This results in better voltage regulation performance with GO-Solar platform (both $30 \%$ and $100 \%$ ).
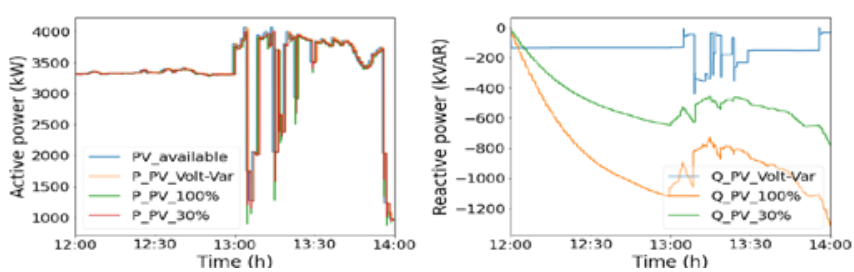

Figure 9. The total active (a) and reactive (b) power of all PV units.

One Simulated PV: To further validate results, one simulated PV is presented in Figure 10. Comparing to the $100 \%$ control scenario, the $30 \%$ scenario has slighter higher curtailment in active power, and larger reactive power output. This can be understood by the fact that each controlled PV needs to contribute more reactive power to regulate system voltage when there are less PVs being controlled. Thanks to the F-OMOO capable of automatically adjusting the reactive power of the controlled PVs to regulate system voltages. The results in Figure 10 provide helpful insights as to how the PVs are controlled differently with fewer PVs contributing reactive power. The results also demonstrate the efficacy of the GO-Solar platform.
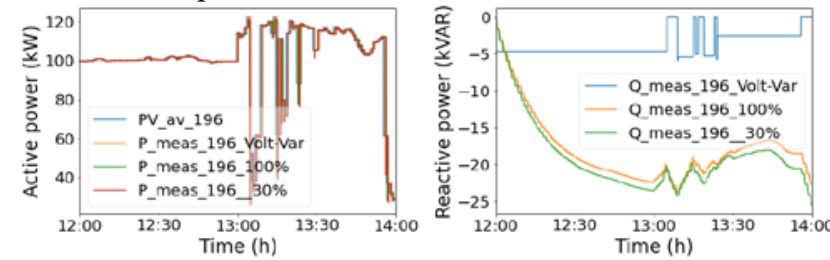

Figure 10. (a) and (b) The active and reactive power of one selected PV.

Performance of State Estimation and OMOO controlling $30 \% \mathrm{PV}$ : The magnitude average percentage error of the state estimation is presented in Figure 11 (a) to show the performance of the state estimation as it predicts the voltage forecasts for the F-OMOO. During the 2-hour run, the error is maintained at less than $0.2 \%$. This indicates high accuracy of the state estimation, which explains why the GOSolar platform can achieve satisfactory voltage regulation performance with only $30 \% \mathrm{PVs}$ being controlled. For the FOMOO, the dual variable, mu, which indicates the voltage regulation progression of the controlled PVs are presented in Figure 11 (b). Some PVs have very small mu values in the beginning and go to zero, while most of PVs have nonzero values during the 2-hour run and continuously regulate PV output active and reactive power.

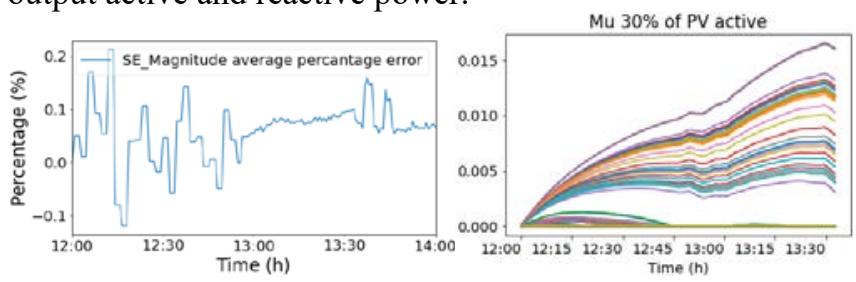

Figure 11. (a) The magnitude average percentage error of state estimation and (b) the dual variables (mu) of F-OMOO.

PHIL Testing Results of controlling 30\% PV: Figure 12 shows the active and reactive power responses for each DER rack. Among the six DER racks, all the PV inverters in the 
second DER Rack are not dispatched by the OMOO agent, and they operate in unity power factor mode. Results show that the closed-loop power outputs at the rest five PCCs track the dispatched power references very closely. In particular, the reactive power at each PCC tracks the dynamics of the references closely even though ripples and noise are observed in multiple PCCs. For the active power output, the transients of the six DER Racks are similar, and Rack \#3-6 have relatively cleaner active power output. For the reactive power, Rack \#1, \#3, \#4 and \#5 have similar responses with reactive power being absorbed all the time, while Rack \#6 has reactive power jumping from absorbing (inductive) to injecting (capacitive) and back repeatedly. The Rack \#2 nominally should not to generate any reactive power, however, noise and harmonics introduce small amounts of reactive power in the simulation. Comparing to the results from the simulated PV, hardware inverters for the Rack \#1, \#4, and \#5 have similar response in active and reactive power.
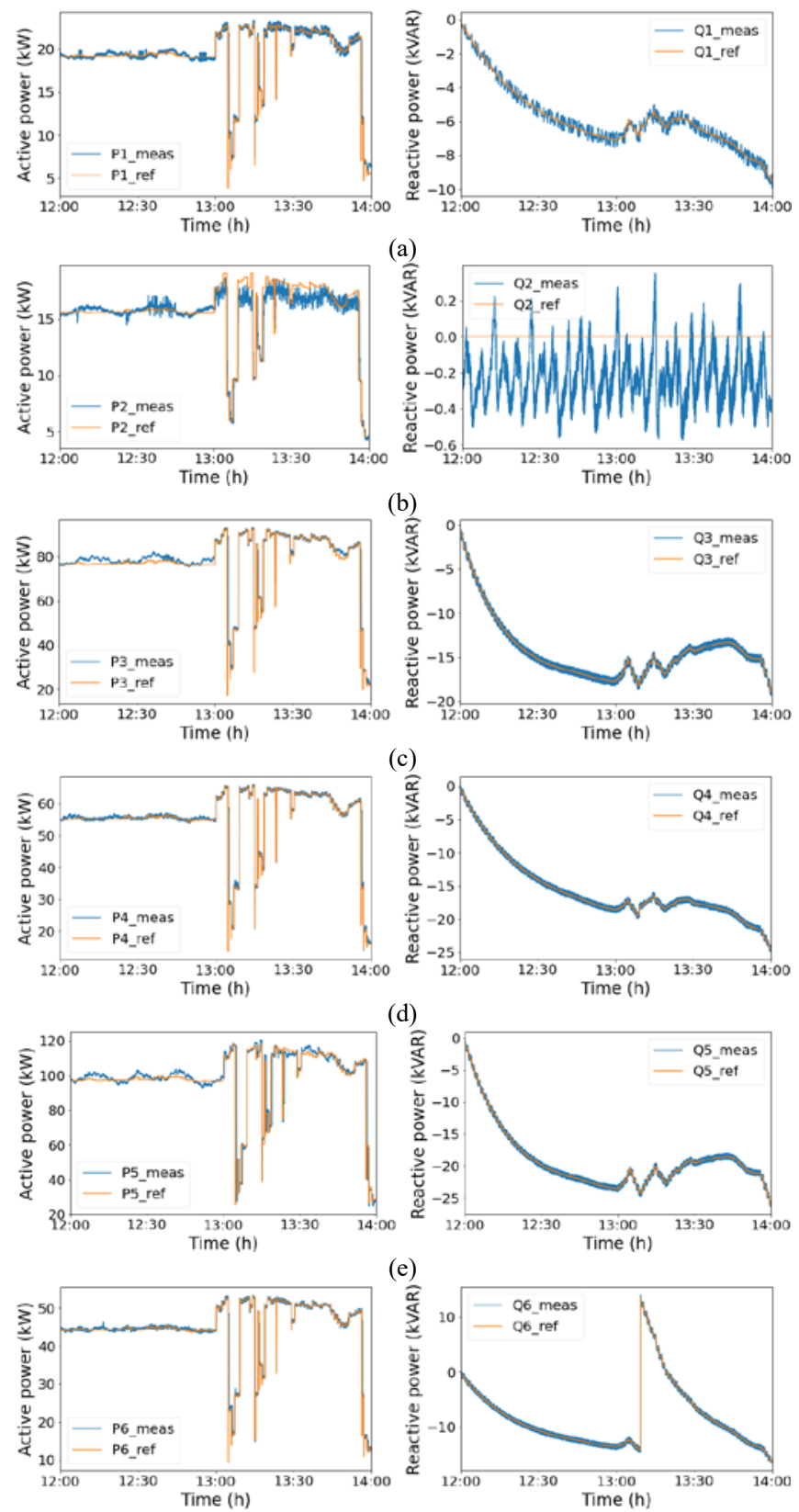

(f)

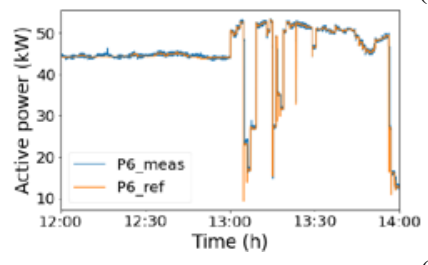

Figure 12. (a)-(f) Experimental results of the the active and reactive power response of each DER rack.
The experimental results in Figure 12 confirms the simulated and hardware inverters work correctly. Overall, the PHIL evaluation results further confirm that: (1) the accurate tracking performance of all DER inverters and racks/PCCs; (2) all inverters can respond fast to the dispatched power set points from the OMOO agent; and (3) the PHIL close-loop with 6 PCCs and the whole platform is stable.

CHIL Testing Results of controlling 30\% PV: Figure 13 shows the CHIL evaluation results of the capacitor bank controller. Figure 13 (a) shows that the control signal and status of the capacitor bank are both " 1 ", indicating the close status. The voltage reference is shown in Figure 13 (b) and its value is within the band (0.95-1.05 p.u.) during the whole test. The two analog input signals, "open" and "close", are presented in Figure 13 (c) and (d) respectively. Both signals are low all the time. Therefore, there is no change in status. These two signals confirm the correct status of the capacitor bank shown in Figure 13 (a). The CHIL evaluation results here further confirm the proper function of the capacitor bank controller and further demonstrates the capability of the GOSolar platform to dispatch the legacy devices and coordinate between slow legacy devices and fast DER inverters.

The HIL evaluation results show that controlling $30 \%$ of PV systems with the GO-Solar platform may provide the best balance of control performance and implementation cost.
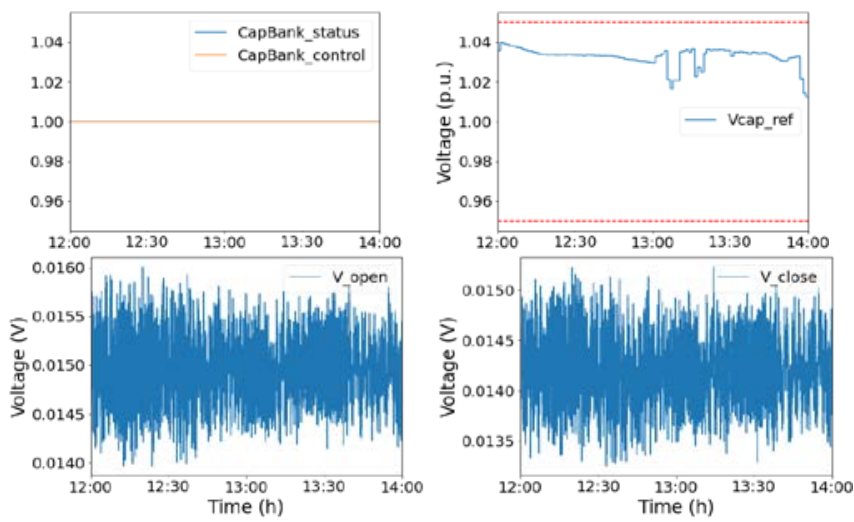

Figure 13. (a) The control signal and measured status of the capacitor bank; (b) the received voltage reference for the capacitor bank controller; and (c) and (d) the open and the close analog signals measured in OPAL-RT.

\section{CONCLUSIONS}

This paper presents the performance evaluation of the GOSolar platform for dispatching slow legacy device(s) and fast DER inverters using an advanced HIL platform. The HIL platform includes co-simulation of the sub-transmission and the complete distribution feeder, the software GO-Solar Platform, power hardware DER inverters, a communications interface, and the capacitor bank controller. HELICS is the key framework used to integrate all the software pieces and hardware devices together and to allow the software controller to interact with the real-time simulation model and hardware inverters as if the controller were interacting with a real-world system. The implementation of such an integrated platform was described with special focus on the HIL setup, CHIL and PHIL implementation. The experimental test with a 2-h simulation of smooth and intermittent solar irradiance demonstrates that the GO-Solar Platform functions well in both clear and cloudy conditions and maintain system voltages within the target limits. The hardware inverters' responses also validate the stability, communications, and functionality of the GO-Solar platform. 


\section{REFERENCES}

[1] Jan Vrins, Global Energy Practice Lead, Distributed Energy Resource, https://www.navigant.com//media/www/site/downloads/energy/2016/ distributedenergyresourcesimpactsonstrategybusines.pdf.

[2] B. Seal, A. Renjit, and B. Deaver, 2018, Understanding DERMS, CA: Electric Power Research Institute.

[3] J. Wang, et al., "Performance Evaluation of Hierarchical Controls for Advanced Distribution Management System-Centered Grid Operations," IEEE PES General Meeting, August 3-6, 2020.

[4] Insights into Advanced Distribution Management System, Voice of Experience, February 2015.

[5] J. Wang, et al., "Performance Evaluation of Distributed Energy Resource Management via Advanced Hardware-in-the-Loop Simulation," IEEE Conference on Innovative Smart Grid Technologies (ISGT), Washington, DC, USA, Feb. 17-20, 2020.

[6] A. Pratt, et al., "A Test Bed to Evaluate Adanced Distribution Management Systems for Modern Power Syystems, " IEEE Eurocon 2019, Novi Sad, Serbia, July 1-4, 2019.

[7] J. Wang, et al., "Hardware-in-the-Loop Evaluation of an Advanced Distributed Energy Resource Management Algorithm," IEEE Conference on Innovative Smart Grid Technologies (ISGT), Feb. 1618, 2021.

[8] B. Palmintier, et al., "Design of the HELICS high-performance transmission-distribution-communication-market co-simulation framework," Workshop on Modeling and Simulation of Cyber-Physical Energy Systems (MSCPES), Pittsburgh, PA, 2017, pp. 1-6.

[9] A Centralized Framework to Enable Visibility and Controllability for $100 \%$ Penetration of Distribution Energy Resources-Grid Optimization with Solar, Y. Zhang, et al., Techinical Report.

[10] J. Wang J Huang, and X. Zhou, "Performance Evaluation of Distributed Energy Resource Management Algorithm in Large Distribution Networks," IEEE PES General Meeting, August 3-6, 2020.

[11] Feeder Voltage Regulation with High-Penetration PV using Advanced Inverters and a Distribution Management System, A Duke Energy Case Study. Bryan Palmintier et al., Technical Report, NREL/TP-5D0065551, Nov. 2016 